\title{
Multifocal Primary Uveal Melanoma: Clinical and Molecular Characteristics
}

\author{
Jose J. Echegaray ${ }^{a}$ Carlos A. Medina ${ }^{a}$ Charles V. Biscotti ${ }^{b}$ Thomas Plesec $^{b}$ \\ Arun D. Singh ${ }^{a}$ \\ ${ }^{a}$ Department of Ophthalmic Oncology, Cole Eye Institute, Cleveland Clinic Foundation, Cleveland, OH, USA; \\ ${ }^{b}$ Department of Pathology, Cleveland Clinic Foundation, Cleveland, OH, USA
}

\section{Established Facts}

- Multifocal primary uveal melanoma is a rare entity with known clinical risk factor of underlying ocular melanocytosis and genetic risk factor with BAP1 mutation.

\section{Novel Insights}

- The clinical and histopathologic characteristics of our two cases suggest two different mechanisms for the development of multifocal uveal melanoma in the absence of BAP1 mutation: de novo growth and malignant transformation of a choroidal nevus.

\section{Keywords}

Choroidal nevus malignant transformation - Germline BAP1 mutation · lodine-125 plaque brachytherapy - Multifocal uveal melanoma

\footnotetext{
Abstract

We report two patients who developed a second distinct choroidal melanoma in the same eye following successful regression of their first choroidal melanoma after iodine-125 plaque brachytherapy. Neither patient demonstrated ocular melanocytosis, local tumor recurrence, or vitreous seeding. One patient had the second tumor arising from a previously
}

documented choroidal nevus, and after undergoing enucleation, there was no detectable connection between the tumors on histopathologic examination. Germline BAP1 mutation was absent in both cases. Multifocal primary uveal melanoma is a rare entity in which the second tumor may occur either de novo or from a malignant transformation of a choroidal nevus. Known risk factors include ocular melanocytosis or germline BAP1 mutation. Additional underlying mechanisms have yet to be elucidated.

(c) 2018 S. Karger AG, Basel

\section{KARGER}

E-Mail karger@karger.com

www.karger.com/oop
(C) 2018 S. Karger AG, Basel Arun D. Singh, MD

Cole Eye Institute, Cleveland Clinic Foundation

2022 E 105th St.

Cleveland, $\mathrm{OH} 44106$ (USA)

E-Mail singha@ccf.org 


\section{Introduction}

Uveal melanoma is the most common primary cancer of the eye in adults, and it almost always presents as a unilateral, unifocal tumor. Bilateral uveal melanoma has been reported, with an estimated lifetime risk of 1 in 50 million, whereas unilateral, multifocal uveal melanoma appears to be even rarer [1-4]. Very few cases of unilateral multifocal uveal melanoma have been reported [57]. Some reported cases occurred in the setting of ocular melanocytosis, which strongly predisposes to uveal melanoma [8]. Herein, we report 2 patients with pathologically confirmed multifocal uveal melanoma that occurred in the absence of ocular melanocytosis, cutaneous melanoma, or familial cancer syndrome [9]. One patient underwent molecular analysis by gene expression profiling of the second tumor, and both underwent BAP1 germline mutational analysis.

\section{Case Report}

Case 1

A 75-year-old white male presented to our clinic 9 years after undergoing iodine-125 plaque brachytherapy with supplemental transpupillary thermotherapy in the right eye for a macular choroidal melanoma extending into the inferotemporal mid-periphery. Treatment and subsequent follow-up had been performed outside our institution. On examination, the tumor remained regressed with no local recurrence (Fig. 1a). However, a distinct pigmented choroidal tumor was detected in the superonasal periphery measuring $8.0 \times 6.5 \mathrm{~mm}$ in basal dimensions and $2.6 \mathrm{~mm}$ in thickness (Fig. 1b). There was an area of normal choroid separating the first and second tumors, with no perceptible connection between them (Fig. 1c). Transscleral fine needle aspiration biopsy of the second tumor confirmed choroidal melanoma (Fig. 1d) with a Class 2 gene expression profile. Sequencing of DNA from peripheral blood lymphocytes revealed no evidence of germline BAP1 mutation (CLIA, Prevention Genetics) to suggest a familial melanoma predisposition. He had no family history of cancer. The patient refused enucleation, so the second tumor was treated with iodine-125 plaque brachytherapy. He died of esophageal carcinoma 19 months after the treatment of the second tumor.

Case 2

A 50-year-old male initially complained of a black spot in his visual field, which led to the discovery of a medium-sized ciliochoroidal malignant melanoma located inferiorly at the 4:30 to 7:00 o'clock position in his right eye. The tumor measured $16.0 \times 14.0$ $\mathrm{mm}$ in basal diameter and $7.5 \mathrm{~mm}$ in thickness and was treated with iodine- 125 plaque brachytherapy, showing tumor regression after 9 years of postoperative follow-up. He was diagnosed with radiation retinopathy and neovascular glaucoma along the course of his follow-up and was treated with panretinal photocoagulation and intravitreal bevacizumab, and a glaucoma drainage implant was placed for intraocular pressure control. On his annual visit 9 years after radiation therapy, his regressed tumor was stable with atrophic margins (Fig. 2a). However, a new melanocytic tumor was identified underlying the macula and extending into the superotemporal mid-periphery, measuring $13.0 \times 12.5 \times 5.3 \mathrm{~mm}$, with associated orange pigmentation and subretinal fluid (Fig. 2b). This tumor corresponded with a previously described choroidal nevus, which had been measured by ultrasonography at $8.5 \times 8.5 \times 2.2$ $\mathrm{mm}$ during the last annual visit (Fig. 2c). Given the patient's poor visual prognosis (longstanding visual acuity of hand motion) and neovascular glaucoma, the patient's eye was enucleated. Histopathology confirmed the diagnosis of a second, independent choroidal malignant melanoma, which showed no anatomic relationship with the initial irradiated melanoma (Fig. 2d). This case of multifocal choroidal melanoma has the unique feature of the second tumor arising from a malignant transformation of a choroidal nevus. Genetic testing showed no germline BAP1 mutation (CLIA, Prevention Genetics). The patient had no relevant family history of cancer.

\section{Discussion}

Our two patients were diagnosed with the second uveal melanoma 9 years after treatment of their initial choroidal melanoma. Clinical features and histopathology indicate that the second lesion did not appear to be continuous with the first melanoma in the patient for which a globe enucleation specimen was obtained for histologic examination. Although it is possible that a single uveal melanoma may be diffuse and multinodular with contiguous spread giving the false impression of multifocal melanoma, this is unlikely to be the case in our patients given the location and time interval between presentations, as the recurrences tend to be marginal and occur within the first 5 years after initial treatment [10]. Furthermore, gonioscopy and histological analysis excluded the possibility of occult ring melanoma or contiguous melanoma [11].

Systemic workup was performed in both cases and did not reveal a non-ocular primary (such as cutaneous or mucosal melanoma) as the source of metastases to the choroid. Another possibility would be that the second tumor is a metastasis of the first tumor. Although metastatic choroidal melanoma to the contralateral choroid has been reported, ipsilateral choroidal metastasis is highly unlikely given the absence of systemic (hepatic) metastasis [12]. In addition, none of our tumors had germline BAP1 mutation [13].

In the past, the presence of oculodermal melanocytosis mainly in Caucasian patients was the only known risk factor for developing multifocal uveal melanoma, with a 30fold greater risk to develop a uveal melanoma than in the general population $[8,14-16]$. None of our patients had 

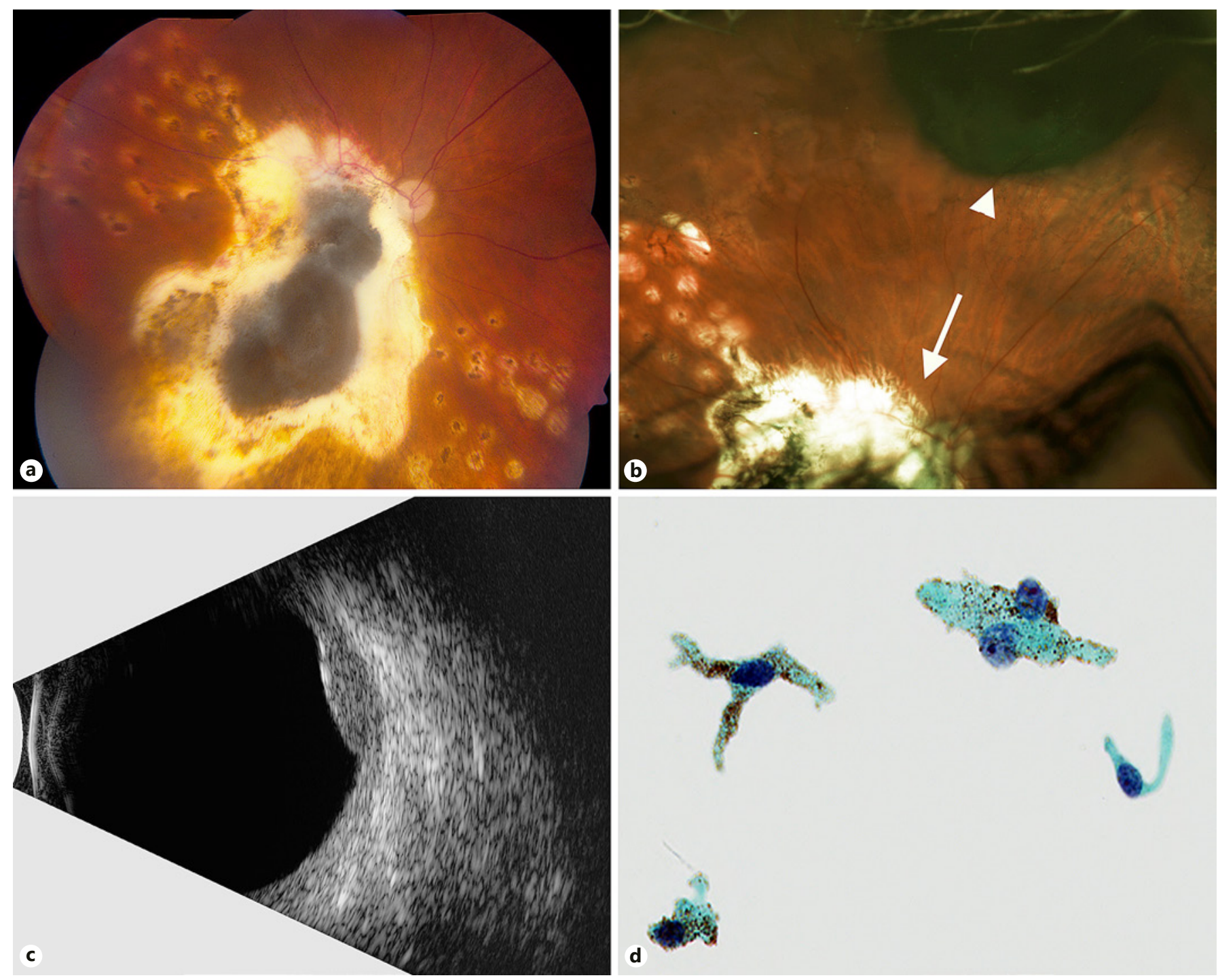

Fig. 1. a A regressed choroidal melanoma 9 years after brachytherapy and transpupillary thermotherapy. Laser photocoagulation scars are observed surrounding the lesion. $\mathbf{b}$ The newly diagnosed pigmented choroidal mass (arrowhead) is observed superonasally in context of a previously treated melanoma (arrow) in the macula. c Longitudinal view of an ultrasonographic B-scan illustrating a dome-shaped choroidal mass of irregular internal structure and sound attenuation (acoustic hollowness), and no extra-

evidence of oculodermal melanocytosis, dysplastic nevus syndrome, or neurofibromatosis type 1 [17]. Additionally, none reported occupational risk factors such as increased UV exposure from arc welding or exposure to industrial toxins $[18,19]$.

A possible clinical risk factor for multifocal uveal melanoma may be the presence of a concomitant cho-

ocular extension. The tumor had low to medium internal reflectivity on diagnostic A-scan (not shown). d The aspirate sample consisted of mostly spindle-shaped cells including some with caudate cytoplasm and finely granular melanin pigment characteristic of uveal melanoma. A minor component of the melanoma cells had an epithelioid appearance characterized by rounded cytoplasmic contours lacking the spindle-shaped processes (Papanicolaou stain, $10 \times$ objective).

roidal nevus at initial presentation that later transformed into a second uveal melanoma, which was the case in one of our patients. Only a limited number of cases have been reported $[5,20,21]$. Such an occurrence would be rare given the low risk of malignant transformation of a choroidal nevus $[5,22]$. The role of radiation exposure from the plaque brachytherapy that delivered 88 Gy to 

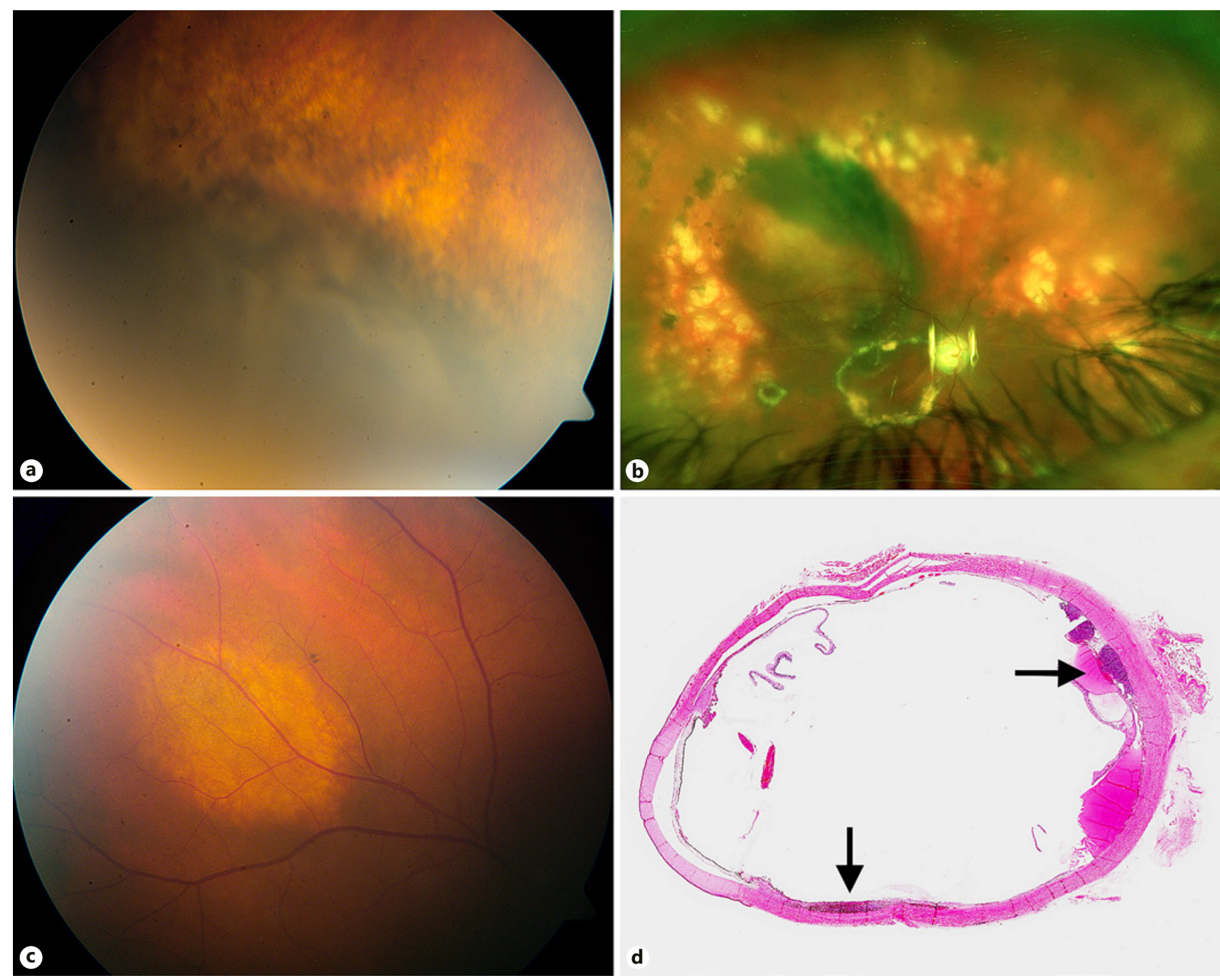

Fig. 2. a Initial choroidal melanoma located inferiorly at the 4:30 to 7:00 o'clock position at the ora serrata with atrophic appearance 9 years after iodine-125 plaque brachytherapy. b Growth of choroidal nevus indicating malignant transformation 9 years after plaque brachytherapy of initial melanoma. c Amelanotic choroidal nevus located superior to the macula 2 years after radiation treatment of his initial melanoma. Surrounding panretinal photoco-

the first tumor located in inferior periphery in inducing malignant transformation of the nevus superotemporal to the macula remains speculative. Using plaque simulator treatment planning software (Plaque Simulator $\mathrm{v}$ 5.3.4; Bebig GmbH, Berlin, Germany) it can be estimated that the location of the nevus received approximately $27 \mathrm{~Gy}$, intervening uvea received more than $27 \mathrm{~Gy}$, and the uvea beyond the nevus received less than $27 \mathrm{~Gy}$, and

yet the second melanoma arose at the site of the preexisting nevus.

The existence of a genetic risk factor for the development of multifocal uveal melanoma can be suspected. In recent years, $B A P 1$ mutation has been suggested as both a risk factor for uveal melanoma metastasis, as well as for the development of uveal melanoma within a germline $B A P 1$ mutation tumor syndrome, which also includes 
mesothelioma, skin melanoma, and renal cell carcinoma [9, 23-25]. Furthermore, the possible relationship between germline $B A P 1$ mutation and multifocal uveal melanoma has been suggested [7]. However, germline BAP1 mutation was absent in both of our cases. Other germline mutations (BRCA2, P16(INK4A), P14(ARF), and P15) associated with uveal melanoma development were not investigated in our cases [26].

The lack of known predisposing clinical or suspected genetic risk factors in our two patients suggests that there are as yet unidentified underlying risk factors for the development of multifocal uveal melanoma that remain to be elucidated. Although the risk of an additional tumor developing in a patient who has already had a primary uveal melanoma is low, careful periodic examination and ultrasonography is required, especially in patients with known coexisting choroidal nevi or oculodermal melanocytosis.

\section{Statement of Ethics}

The study complied with the guidelines for human studies and animal welfare regulations. The subject gave informed consent and the study protocol was approved by the institute's committee on human research.

\section{Disclosure Statement}

The authors have no relevant financial disclosures.

\section{References}

1 Blumenthal EZ, Pe'er J: Multifocal choroidal malignant melanoma: at least 3 melanomas in one eye. Arch Ophthalmol 1999;117:255-258

2 Singh AD, et al: Bilateral primary uveal melanoma. Bad luck or bad genes? Ophthalmology 1996; 103:256-262.

-3 Volcker HE, Naumann GO: Multicentric primary malignant melanomas of the choroid: two separate malignant melanomas of the choroid and two uveal naevi in one eye. $\mathrm{Br}$ Ophthalmol 1978;62:408-413.

4 Shammas HF, Watzke RC: Bilateral choroidal melanomas. Case report and incidence. Arch Ophthalmol 1977;95:617-623.

5 Filloy A, et al: Cytogenetical study of a multifocal uveal melanoma. Semin Ophthalmol 2014;29:163-165.

-6 Dithmar S, Volcker HE, Grossniklaus HE: Multifocal intraocular malignant melanoma: report of two cases and review of the literature. Ophthalmology 1999;106:1345-1348.

7 Rao R, et al: Multifocal choroidal melanoma in a patient with germ line BRCA-associated protein 1 mutation. Retin Cases Brief Rep 2018;12:1-4.

8 Singh $\mathrm{AD}$, et al: Lifetime prevalence of uveal melanoma in white patients with oculo(dermal) melanocytosis. Ophthalmology 1998;105:195198.

-9 Testa JR, et al: Germline BAP1 mutations predispose to malignant mesothelioma. Nat Genet 2011;43:1022-1025.
10 Bellerive C, et al: Local failure after episcleral brachytherapy for posterior uveal melanoma: patterns, risk factors, and management. Am J Ophthalmol 2017;177:9-16.

11 Hammer ME, Margo CE: Unilateral multifocal uveal melanoma with occult ring melanoma. Arch Ophthalmol 2002;120:1090-1091.

12 Singh $\mathrm{AD}$, et al: Choroidal melanoma metastatic to the contralateral choroid. Am J Ophthalmol 2001;132:941-943.

13 Harbour JW, et al: Frequent mutation of BAP1 in metastasizing uveal melanomas. Science 2010;330:1410-1413.

14 Honavar SG, et al: Two discrete choroidal melanomas in an eye with ocular melanocytosis. Surv Ophthalmol 2002;47:36-41.

15 Gonder JR, et al: Uveal malignant melanoma associated with ocular and oculodermal melanocytosis. Ophthalmology 1982;89:953960.

16 Velazquez N, Jones IS: Ocular and oculodermal melanocytosis associated with uveal melanoma. Ophthalmology 1983;90:1472-1476.

17 Friedman SM, Margo CE: Choroidal melanoma and neurofibromatosis type 1 . Arch Ophthalmol 1998;116:694-695.

18 Albert DM, Puliafito CA: Choroidal melanoma: possible exposure to industrial toxins. $\mathrm{N}$ Engl J Med 1977;296:634-635.
19 Turaka K, et al: Bilateral uveal melanoma in an arc welder. Graefes Arch Clin Exp Ophthalmol 2011;249:141-144.

20 Spraul CW, Gackle HC, Lang GK: Malignant transformation of a choroidal nevus 6 years after brachytherapy of a non-contiguous choroidal melanoma - a case report and review of literature (in German). Klin Monbl Augenheilkd 2001;218:626-631.

21 Fabian ID, Arora AK, Cohen VM: Malignant transformation of a choroidal nevus in an eye treated for choroidal melanoma. Retin Cases Brief Rep 2017;11:131-133.

22 Singh AD, Kalyani P, Topham A: Estimating the risk of malignant transformation of a choroidal nevus. Ophthalmology 2005;112:17841789.

23 Abdel-Rahman MH, et al: Germline BAP1 mutation predisposes to uveal melanoma, lung adenocarcinoma, meningioma, and other cancers. J Med Genet 2011;48:856-859.

24 Wiesner T, et al: Germline mutations in BAP1 predispose to melanocytic tumors. Nat Genet 2011;43:1018-1021.

25 Popova T, et al: Germline BAP1 mutations predispose to renal cell carcinomas. Am J Hum Genet 2013;92:974-980.

26 Hearle N, et al: Contribution of germline mutations in BRCA2, P16(INK4A), P14(ARF) and P15 to uveal melanoma. Invest Ophthalmol Vis Sci 2003;44:458-462. 